\title{
LA BELLE ET LA BÊTE, OU COMMENT LA BELLE EST DEVENUE PRINCESSE...
}

\author{
Ève Gardien \\ Sociologue, directrice du Forum \\ "Vie professionnelle " du collectif Reliance
}

L'exploration sociologique du conte de fée « La Belle et la Bête » amène l'auteur à proposer une interprétation de ce récit complémentaire à celle - déjà très connue - produite par le psychanalyste Bruno Bettelheim. II ne s'agit plus de la lente transformation de la Bête en un beau prince charmant, mais bien d'un changement réciproque de la Belle et de la Bête, l'une en princesse et l'autre en prince. Les enjeux du regard d'autrui dans la construction identitaire d'un individu sont ainsi dévoilés.

« II était une fois... » Une fois invoqué, ce sésame magique de l'enfance nous introduit à l'œuvre cinématographique La Belle et la Bête de Jean Cocteau ', et nous dévoile un monde sans féerie : un univers fondé sur les apparences, dans lequel seule l'expérience du Beau fascine.

Une flèche décochée par un archer maladroit introduit notre regard à l'intérieur de la pièce où les deux sœurs terminent leurs préparatifs en vue de la soirée chez la duchesse. Elles sont toutes deux à leurs atours, rivalisant d'élégance et convaincues qu'une apparence

I. J. Cocteau, La Belle et la Bête, film noir et blanc, présenté au festival de Cannes en 1946, a obtenu le prix Louis Delluc. Le scénario est de Jean Cocteau, d'après le conte de Mme Leprince de Beaumont. 
bien soignée est le signe distinctif d'une multitude de qualités positives et d'une certaine noblesse.

De fait, dans ce monde, tous les personnages adhèrent et contribuent à cette valorisation de l'esthétique. Par exemple, les jeunes hommes accordent un intérêt certain à la Belle en raison de sa plastique. La fascination exercée sur ces jeunes hommes est telle que l'un d'eux, Avenant, ne respecte qu'à grand-peine le refus que lui oppose la Belle de se marier avec lui. Quant aux sœurs, elles escomptent faire un bon mariage grâce à leurs apparats, nous rappelant les liens socialement construits entre beauté et statut social: «... une extrême beauté peut apparaître comme un vaste privilège de classe, réservé aux femmes d'un très haut niveau socio-économique ${ }^{2}$. " Ces deux sœurs estiment d'ailleurs devoir être traitées comme des grandes de ce monde et s'indignent du peu de diligence dont font preuve leurs laquais à leur égard. Toujours, selon cette logique sociale distinguant les uns et les autres en fonction de l'apparence, les sœurs traitent la Belle, qui n'accorde que peu de cas à son apparence, comme une servante. Autre exemple, le frère raille la vanité de ses sœurs, il rit de leur prétention à vouloir paraître plus que ce qu'elles sont. Enfin, pour ce qui concerne la Belle, bien que le film souligne longuement et à plusieurs reprises les qualités de son cœur, nous pouvons constater qu'elle a aussi intériorisé et accepté cet ordonnancement social fondé sur la valorisation de la beauté. Son statut de servante est justifié à ses yeux par la beauté de ses sœurs et la blancheur de leurs mains.

Ainsi, tout ce monde est régenté par un ordre, distinctif et distinguant, reposant sur les apparences esthétiques des personnages. Et quand un certain désordre surgit, il provient non pas d'une remise en cause de cette valorisation de l'esthétique mais bien d'une estimation individuelle divergente de la beauté. Le traitement d'une personne apparaît dès lors comme étant, pour partie, le résultat du regard posé sur son apparence, la conséquence d'une estimation sociale de sa valeur en fonction de ce qu'elle présente d'elle-même. Jean Baudrillard souligne le lien entre cet univers pourtant imaginaire et notre monde contemporain ${ }^{3}$. Cet essai cinématographique nous convie donc à une réflexion sur les significations véhiculées en lieu et place de notre regard : le réel n'apparaît pas tel qu'il est mais tel que nous le percevons. II en est ainsi pour les personnes que nous côtoyons. Notre regard les crée dans la mesure où il leur donne sens et leur attribue une valeur. Et ce jugement ne saurait être détaché de toute influence sociale ${ }^{4}$.

C'est bien à une réflexion sur notre regard à laquelle nous sommes conviés à travers cet essai cinématographique. II s'agit là d'une réflexion sur les interactions entre un regard porté sur autrui, le traitement social qui en découle et la construction identitaire de la personne jaugée ${ }^{5}$.

Après une présentation de ce monde, l'action débute : le père, en route pour sa maisonnée, perd son chemin et pénètre alors dans un nouvel univers, un « autre » monde ordonné par des règles magiques, un lieu étrange et étranger où la Bête vit en recluse. Un lieu étrange et étranger? En effet, ce monde est une remise en cause de nos habitudes d'agir et de pensée. II s'agit d'un autre univers, régi par d'autres lois sous le regard omniprésent de nombreuses statues de marbre muettes, un lieu où l'expérience de l'étrangeté est quoti-

\footnotetext{
$\gg>$

2. J. Maisonneuve et M. Bruchon-Schweitzer (198I), Modèles du corps et psychologie esthétique, Paris, PUF.

3. « Ce procès de différenciation statutaire est un procès social fondamental, par où chacun s'inscrit en société [...] c'est l'inscription permanente dans un code dont les règles, les contraintes de signification - comme celles de la langue - échappent pour l'essentiel aux individus », J. Baudrillard (1970), La société de consommation, Paris, Denoël, p. 79.

4. «L'accentuation de l'homogénéité des jugements avec l'âge plaide en faveur de l'hypothèse d'une inculcation sociale de modèles stéréotypés », J. Maisonneuve et M. BruchonSchweitzer (198I), Modèles du corps et psychologie esthétique, Paris, PUF.

5. « Le corps socialement objectivé est un produit social qui doit ses propriétés distinctives à ses conditions sociales de production et le regard social n'est pas un simple pouvoir social et abstrait d'objectivation, comme le regard sartrien, mais un pouvoir social qui doit toujours une part de son efficacité au fait qu'il trouve chez celui auquel il s'applique la reconnaissance des catégories de perception et d'appréciation qu'il lui applique », P. Bourdieu (1977), « Remarques provisoires sur la perception sociale du corps », Actes de la Recherche en Sciences Sociales, $n^{\circ} 14$
} 
dienne, un espace où un autre regard devient possible par l'expérimentation d'une nouvelle normalité.

Alors que le père cueillait une rose blanche en pensant à la requête de sa plus jeune fille, il découvre avec effroi qu'il a enfreint la seule règle, l'unique restriction à sa jouissance de ce lieu magique: la Bête le condamne à mort. Que protège cet interdit dont la transgression est ainsi punie? Quelle symbolique se cache derrière cette rose blanche qu'il ne fallait pas cueillir? À l'instar du cheval blanc nommé le Magnifique, ou encore des mains trop blanches pour travailler, cette rose blanche représente la Beauté. L'unique chose visà-vis de laquelle la Bête marque un attachement tout particulier, et pour laquelle elle ressent un violent sentiment de perte, est précisément ce qui ne participe pas à son être : la beauté.

Ainsi, il apparaît que la vie de la Bête est aussi organisée autour de la valeur structurante de la Beauté. Cette dernière est d'ailleurs la raison de son exil hors du monde des humains, et probablement aussi la raison de son refus d'être traitée comme un « Monseigneur ». Comment pourrait-elle être un «Monseigneur »? En effet, la Bête n'est pas indemne des regards pleins d'effroi et de terreur qu'elle provoque. Son identité a pris sens à ses yeux dans le regard d'autrui, regard empli d'horreur, regard la rejetant hors de son humanité et l'instituant dans sa monstruosité. L'intensité de cette réaction de dégoût, que sa seule vue provoque, souligne l'importance extrême accordée à l'esthétique dans l'attribution d'une identité par et pour autrui. «La présence esthétique est [...] la somme de toutes les composantes identitaires repérables, être homme ou femme de tel ou tel âge, d'ici ou de là, de tel ou tel milieu, etc., fondues dans une harmonie de couleurs, de traits et de postures; c'est lui,

$>>$

6. V. Nahoum-Grappe (1988), «Regards croisés sur la différence : l'esthétique du corps », Sociétés, n 21 , p. 22.

7. Lire à ce sujet : E. Goffman, 1975 (1963 pour la version originale américaine), Stigmate - les usages sociaux des handicaps, Paris, Minuit. c'est elle ! L'énigme esthétique pose et résout la question de l'identité de l'Autre ${ }^{6}$. $/$ La Bête s'est identifiée au jugement porté sur elle : une " bête ".

C'est pourquoi la Bête est devenue cet être mi-homme mi-animal, cet être au corps humain et à la pilosité animale, profondément marqué par une ambivalence identitaire douloureuse, preuve même de l'identification de la Bête au stigmate ${ }^{7}$, dont la Belle devine le sens caché : « une moitié de lui est en lutte contre l'autre ». Son humanité et sa monstruosité tour à tour, ou en même temps, se rejettent. Toute l'ambivalence identitaire de la Bête se résume en ce qu'elle pense appartenir à l'espèce humaine et en espère alors la considération qui en découle, et en ce qu'elle admette, à d'autres instants, son apparence bestiale, acceptant par conséquent la façon dont elle est regardée et rejetée. Et cette double perspective sur elle-même s'inscrit dans ce que la Bête donne à voir d'elle. La Belle dira à son père : « Parfois il a une démarche royale! Parfois, il boîte presque, il semble victime de quelque infirmité... »

La Bête est le fruit de ce double regard sur elle-même : à la fois elle estime que son cœur est bon et revendique alors une certaine noblesse, à la fois elle constate sa laideur apparente et se sent accablée par les implications sociales qui en découlent. En intériorisant le système de valeurs mondaines, la Bête a appris à ressentir de la honte vis-à-vis d'elle-même, peut-être même du mépris ou de la haine. Cependant, cette transmission culturelle lui a de plus enseigné l'importance d'autres qualités, dont elle est détentrice, et qui, si elles n'étaient pas négligées du fait du stigmate, ni évincées par un attachement certain du regard aux apparences physiques, pourraient lui permettre d'escompter un traitement social tout autre. Ainsi, il apparaît que le regard est sélectif : valorisant certains aspects, en négligeant d'autres. Ce regard est empreint de partialité : regard partiellement aveugle ou regard défigurant autrui par la sélection de traits qu'il opère?

Le conte de fée va répondre à cette question : la Belle va être contrainte à un temps hors du monde réel, à une expérience de vie partagée 
dans la durée aux côtés de la Bête. Cette opportunité d'expérimenter un quotidien commun avec la Bête se présente comme un emprisonnement cruel de la Belle. Mais une telle expérience aurait-elle pu exister sans une certaine coercition? II faut bien admettre que cette nouvelle enculturation ne pouvait se dérouler dans le monde ordinaire, monde à l'intérieur duquel l'évidence - toute culturelle de l'impossibilité d'un lien humain et constructif avec la Bête est solidement ancrée dans les regards: la monstruosité est chose vécue comme naturellement effrayante, et cela sans égard pour sa relativité culturelle. En effet, prenons un exemple soulignant cette relativité culturelle: que dire de l'exposition ${ }^{8}$ des enfants avec un «bec de lièvre » en Grèce antique ? Cette pratique socialement légitimée en Grèce antique semblerait aujourd'hui, si elle se produisait, un véritable acte de barbarie.

C'est cette vie commune sur un long temps qui favorisera le développement progressif d'un discernement inhabituel vis-à-vis de la Bête jusqu'à aboutir à une véritable mutation du regard de la Belle, à une connaissance au plus près de la singularité de la Bête. La Belle découvre tout ce que son regard ne pouvait capter au premier coup d'œil - l'individualité de la Bête - et c'est dans la reconnaissance de la différence constituante de chacun que la Belle découvre progressivement un être bon et patient, un être s'intéressant à son bien-être et lui offrant de somptueux cadeaux, un être qui ne lui tient pas rigueur de sa frayeur initiale et prend le temps de l'apprivoiser, enfin, et avant tout, un être qui la traite comme une princesse.

Ainsi, la Belle expérimente un nouveau regard sur sa propre personne, une nouvelle manière d'agir envers elle pleine de tact et de prévention. De fait, la Bête refuse systématiquement d'instaurer un rapport générant une attitude de soumission de la Belle à son geôlier, mais, bien au contraire, elle s'enquiert de ses désirs et de ses souhaits : " II n'y a ici de maître que vous... ». Même la volonté de la Belle de rejoindre son père mourant est entendue et respectée par la Bête, alors qu'il en va de sa vie. Cette relation de bientraitance favorise peu à peu un changement identitaire de la Belle, malgré une forte réti- cence initiale: " Je ne me sens pas à l'aise dans ces beaux atours. Et je n'ai pas l'habitude que l'on me serve. » La Belle devient progressivement princesse.

Tant et si bien que, lorsqu'elle retourne visiter son père dans le monde réel, il lui est très douloureux d'être remise dans le rôle de servante des membres de sa famille. Elle a intériorisé un autre regard sur elle et pour cette raison escompte une autre façon de se comporter vis-à-vis d'elle. D'ailleurs, dès qu'elle est seule dans sa chambre, à l'abri des railleries de ses sœurs, elle continue à se parer comme une princesse. La Belle s'est identifiée au regard que porte la Bête sur elle, et aspire à être traitée avec respect et bonté.

Aucun personnage ne peut alors rivaliser avec cette relation qui apporte un tel épanouissement à la Belle. Même l'homme fringuant qu'est Avenant, tout en déclarations d'amour et demandes en mariage, n'est pas capable de la regarder comme une princesse : il essaye de lui imposer ses désirs, estimant par là vouloir son bien. L'expérience de la relation à la Bête est la révélation pour la Belle de ce qu'elle peut devenir : un monstre si elle laisse la Bête mourir de chagrin, une princesse si elle reste sous son regard. En fin de compte, cette expérience auprès de la Bête favorise l'acquisition par la Belle de nouveaux repères et d'une nouvelle compréhension. La confrontation durable à cet univers magique a permis l'ouverture de la Belle à une certaine liberté : la relativisation de ses habitudes de pensée et l'émergence d'un nouveau regard sur elle-même.

Parallèlement, la Belle change son regard sur la Bête et cela ouvre un nouveau champ de possibles relationnels entre elle et la Bête. En effet, tant que la Belle imaginera que la Bête la traite comme une princesse uniquement pour lui plaire et la séduire, la Bête restera prisonnière de son apparente animalité. La Bête est alors non seulement vue mais aussi comprise à travers sa hideur. Sa monstruosité est la catégorie

8. Cf. H.-J. Sticker (1997 [1982]), Corps infirmes et société, Paris, Dunod, chapitre III. 
générique à travers laquelle tout son être est subsumé. Et lorsqu'une certaine beauté émane d'elle, elle s'explique alors comme une tentative de compensation de sa hideur : « Mais je devine que vous faites l'impossible pour essayer de me faire oublier votre laideur ! ». Et effectivement, aussi longtemps que la Belle focalisera son regard uniquement sur la monstruosité de la Bête, oublier sa laideur sera impossible.

Cependant, graduellement, une connaissance plus aboutie, développée dans un quotidien partagé, permet à la Belle de dompter son dégoût et de passer outre ses peurs initiales. Nous sommes alors loin de la Bête observée à son insu, Bête avec des mains fumantes suggérant un meurtre encore frais. La Bête est devenue celle qui lape paisiblement l'eau de l'étang pour se désaltérer. Bien évidemment, la Bête est encore vue au travers de son animalité apparente, mais cet apaisement du regard de la Belle l'amène à pouvoir proposer à la Bête une promenade commune dans le jardin. II devient possible de partager une expérience sans que la rencontre des deux regards soit une source d'effroi. La Bête semble d'ailleurs plus douce à la Belle, est-ce d'être traitée autrement? Est-ce d'être regardée autrement? Difficile de trancher et la Belle hésite : " on dirait que vous avez la voix plus douce... » La Bête serait-elle moins animale qu'elle ne le paraît?

Au fil de leur relation, la Belle décille son regard, et finira par refuser à la Bête la possibilité de se réfugier derrière l'explication facile de son animalité apparente pour implorer le pardon d'un écart de conduite : « Ces paroles vous vont aussi mal que possible ! N'avez-vous pas honte! Nettoyez-vous! ॥ La Bête n'est plus seulement animale aux yeux de la Belle : elle en exige alors de nouveaux comportements relatifs à son humanité. Ce regard de dégoût de la Belle sur les actions jugées " animales» de la Bête provoque alors une réaction violente chez cette dernière qui supplie, désemparée: «Fermez votre porte! Vite ! Vite ! Fermez votre porte ! Votre regard me brûle ! Je ne supporte pas votre regard ! » Le nouveau regard porté par la Belle sur la Bête, un regard qui lui impose d'être à la hau- teur de son humanité est insupportable pour la Bête, probablement à la fois parce qu'elle est surprise de ces nouvelles attentes vis-à-vis d'elle, et d'autre part parce que ce qu'elle espérait - être considéré comme un humain et traité comme tel - comporte un ensemble d'exigences auxquelles elle n'a pas encore été concrètement confrontée et qui nécessite un apprentissage tout nouveau de sa part : il lui faut développer son potentiel d'humanité. La Bête peut donc enfin devenir humaine grâce à l'espace relationnel nécessaire à cette transformation qui vient de s'ouvrir dans le regard de la Belle. Cette dernière observe la beauté de son cœur au même titre que son apparence disgracieuse : « ce monstre est bon », dira-t-elle à son père.

Cependant, le rempart de la monstruosité reste bel et bien dressé entre la Belle et la Bête, et même sa bonté maintenant reconnue n'est pas suffisante pour faire de la Bête un être digne d'amour. À la fin du film, la Bête se meurt au fond du jardin magique, désespérée d'être sans nouvelles de la Belle: c'est alors que le miroir magique se brise en morceaux. La Belle mesure à cet instant toutes les conséquences de son absence pour la Bête et se précipite à sa recherche dans le monde magique. Elle découvre la Bête mourant de chagrin et d'affliction, couchée dans les hautes herbes, et la supplie de revenir à la vie. La Bête lui répond: "Belle, si j'étais un homme, sans doute je ferais les choses que vous me dites. Mais les pauvres Bêtes, qui veulent prouver leur amour, ne savent que se coucher par terre et mourir... ॥ C'est devant la mort imminente de la Bête que la Belle pourra enfin la regarder avec amour et la délivrer définitivement de son animalité. La Bête se transformera alors en beau prince charmant.

Que nous apprend ce conte de fées ? Faire naître un nouveau regard est un processus qui nécessite du temps, le temps d'un apprivoisement réciproque pour le partage d'une re-connaissance commune, le temps d'une expérience quotidienne pour dépasser la culture initialement transmise. La première fois que la Belle aperçoit la Bête, elle s'évanouit de frayeur, alors qu'à la fin du film, elle déclare: «j'aime avoir peur... avec vous! » 
En fin de compte, cette histoire pour enfants nous raconte comment la rencontre entre deux êtres peut devenir une transformation mutuelle par un changement réciproque de regard. En ce sens, modifier son regard sur autrui revient à lui offrir un espace de liberté dans lequel il peut devenir un autre lui-même. Accepter de changer de regard revient à donner à l'autre l'opportunité de développer un potentiel jusqu'alors resté en jachère et à l'autoriser à se révéler prince ou princesse. Même si le sort de la Bête peut sembler ne pas nous concerner du fait de sa différence corporelle manifeste et initiale, nous découvrons cependant que le processus social à la genèse des identités est à l'œuvre dans la vie de chacun d'entre nous. II peut même invalider les plus beaux d'entre nous : la Belle se prenait pour une servante. 